\title{
Abnormal U Waves in the Electrocardiogram in Subarachnoid Haemorrhage
}

\author{
J. DE SWIET
}

\author{
From East Glamorgan District General Hospital, Pontypridd, South Wales
}

Numerous references to abnormal electrocardiograms in subarachnoid haemorrhage have appeared in recent years, describing changes in the $S-T$ segment, $Q-T$ interval, $T$ wave, and positivity of the $U$ wave. Abnormalities of the $U$ wave appear to have been the least pronounced and confined to "prominence" or "positivity". In the case described below, independent deeply negative $U$ waves and a prolonged $Q-U$ interval are shown, apparently for the first time.

\section{Case Report}

A woman of 71 was admitted to hospital with a history of the sudden onset, 3 hours earlier, of headache and loss of consciousness which had improved to drowsiness by the time of admission. The previous history was of a total mastectomy 11 years before, and of herniotomy 2 months before admission. On examination, the blood pressure was $200 / 100 \mathrm{~mm}$. $\mathrm{Hg}$, temperature $35.5^{\circ} \mathrm{C}$., pulse 80 , respirations 20 . All tendon reflexes were brisk and plantar responses were bilaterally extensor. There was moderate neck rigidity; pupils were equal and reacted to light, and there was a small left subhyaloid haemorrhage. There was a grade $4 / 6$ pansystolic murmur at the mitral area, conducted well out to the axilla and accompanied by a thrill. Lumbar puncture showed uniformly blood-stained fluid at a pressure of $80 \mathrm{~mm}$. The next day coma deepened and continued until her death 8 days after admission.

Investigations, the day after she was admitted, showed: serum electrolytes: $\mathrm{Na} 138, \mathrm{~K} 4 \cdot 2$, and $\mathrm{Cl} 95$ $\mathrm{mEq} / 1$; ; blood sugar $104 \mathrm{mg}$./100 ml., urea $41 \mathrm{mg}$./100 $\mathrm{ml}$., $\mathrm{Hb} 14 \mathrm{~g}$./100 ml., leucocytes 15,000/cu.mm., with normal differential, erythrocyte sedimentation rate (Westergren) $22 \mathrm{~mm}$. in first hour. The electrocardiogram at this time (Fig. 1) showed sinus rhythm, rate 82, left axis deviation, and diphasic $T$ wave in lead $I$. In the praecordial leads V2 showed a raised S-T segment and deeply negative $U$ wave, V3 a wider and more deeply $(2 \mathrm{~mm}$.) negative $U$, which became progressively more shallow towards V6 and allowed the previously "submerged" $P$ waves to appear. The Q-U interval was $0.64 \mathrm{sec}$. (normal $0.58 \mathrm{sec}$. (Lepeschkin, 1955)). The S-T segment was raised from V3 to V5 inclusive, and very slightly depressed in V6. The $T$ wave was flat in V4, negative in V5, and diphasic in V6.

Two days later the serum electrolytes were: $\mathrm{Na} \mathrm{135,}$ $\mathrm{K}$ 4.6, and $\mathrm{Cl} 95 \mathrm{mEq} / \mathrm{l}$. Six days after admission the electrocardiogram (Fig. 2) showed taller $P$ waves (2.5 $\mathrm{mm}$.) in leads II and VF, a "staircase" S-T segment in leads II, III, and VF, isoelectric S-T segments in the praecordial leads, and much smaller $U$ waves from V3 to V6. The rate was 98 and the Q-U interval was 0.52 sec. against an expected $0.5 \mathrm{sec}$.

At necropsy the heart weighed $325 \mathrm{~g}$. The myocardium was macroscopically normal throughout except for a tiny pale area in the upper septum, and some fibrosis at the tips of the papillary muscles of the mitral valve. There was considerable calcification of the mitral valve ring, with a little thickened fibrosis of the cusps and shortening of the chordae. The aortic cusp fibrosis and valve ring calcification produced narrowing of the outflow tract, resulting in thickening of the endocardium locally but without involving the underlying myocardium. There was slight patchy atheroma in the right coronary artery and in the circumflex branch of the left, with minimal atheroma in the anterior descending branch. There were multiple ante-mortem thrombi in the branches of the pulmonary artery to the lower lobe of the right lung, probably derived as emboli from thrombus found in the left femoral and saphenous veins, which were recent and probably responsible for multiple small peripheral red infarcts in the right lower lobe.

There was extensive recent subdural and subarachnoid haemorrhage, mostly left-sided and over the left cerebral hemisphere. There was evidence of rupture of a large congenital aneurysm at the junction of the left internal carotid artery and the left posterior communicating artery, and considerable recent softening of the left uncal, hippocampal, and basal ganglia regions. There was no intracerebral haemorrhage, and no other aneurysms were found.

Extensive histological examination of the myocardium showed pronounced changes in the pale area of the upper 

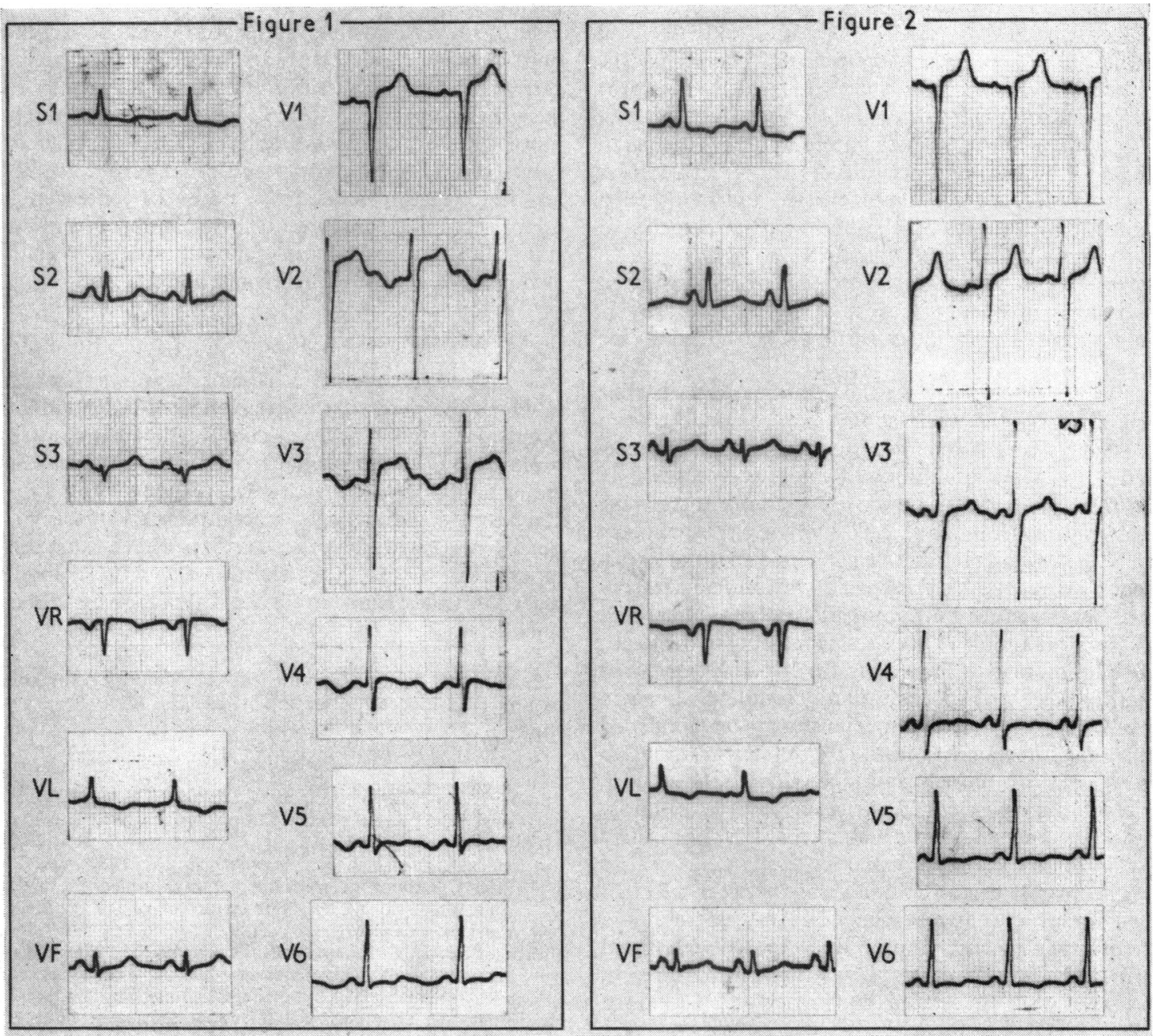

Fig. 1.-Electrocardiogram one day after admission, showing deeply inverted $U$ waves in V2 to V5 and very prolonged Q-U, with raised S-T segments in V1-V5.

FIG. 2.-Electrocardiogram 5 days later, showing taller $P$ waves in $S 2$ and VF, and a "staircase" $S-T$ segment in S2, S3, and VF. S-T segments are isoelectric in V3-V6 and the $U$ waves are much smaller. The $Q-U$ interval is almost normal and so all $P$ waves appear.

septum, in an area of the right ventricle adjacent to the septum posteriorly, in the papillary muscles of the mitral valve, and in the subendocardial areas of the ventricles adjacent to the zones thus involved. The main abnormality was microscopical focal fibrosis, often associated with increased vascularity, and in some cases pleomorphism of muscle fibre and nuclear size, and fuchsinophilic staining reaction. These changes suggested recent as well as previous myocardial damage, but none were of an inflammatory nature, and were considered compatible with longstanding focal ischaemic myocardial lesions. However, the coronary arteries and arterioles only showed some medial hypertrophy.

\section{Discussion}

Burch, Meyers, and Abildskov (1954) reviewed the electrocardiograms in 17 cases of cerebral vascular catastrophes, in all of which the $U$ waves were described as "present" or "not prominent"; while the Q-T interval in subarachnoid haemorrhage appears to be variable, in that Shuster $(1960)$ found a short Q-T, and he agreed with Burch et al. that they probably measured the Q-U. Hoffbrand and Morgan (1965) found a prolonged Q-T, probably attributable to a very wide negative $T$ wave repre- 
senting T-U fusion, as in the cases described by Wasserman et al. (1956), Koskelo, Punsar, and Sipila (1964), and Srivastava and Robson (1964). However, Hugenholtz (1962) stressed a real prolongation of $\mathrm{Q}-\mathrm{T}$.

Negative $U$ waves may rarely appear as isolated normal findings, and abnormally in left ventricular hypertrophy, angina, hypertension, and myocardial infarction. Negative $U$ waves in subarachnoid haemorrhage, occurring distinct from negative $T$ waves, do not appear to have been demonstrated previously, probably because of the hitherto invariably seen $\mathrm{T}-\mathrm{U}$ fusion postulated by authors (see above).

Papp (1940) stated that the idea of an extracardiac origin of the $U$ wave was untenable; and Furbetta et al. (1956) categorically concluded that they specified all possible alterations of the $U$ wave and T-U segment, and that they were present only and always with pathological conditions involving the papillary muscles. While it must be conceded that the "residual" changes still present in the second electrocardiogram could be due to the macroscopical and histological abnormalities described above, the death of the patient two days after this tracing prevented the possibility of recording any complete return to normality. It must also, however, be conceded that the abnormalities in the first electrocardiogram were so grossly more pronounced than in the second, and the improvement in them so rapid, as to imply superimposed temporary abnormalities not solely explicable by the myocardial lesions.

The problem of distinguishing between electrocardiographic changes solely due to subarachnoid haemorrhage, and those due to subarachnoid haemorrhage with concomitant myocardial disease, has been well illustrated by Sarner and Crawford (1965). They found a "gross excess" of coronary occlusion at necropsy in men aged 45-69, dying from ruptured intracranial aneurysms, in that 18 out of 102 such patients had complete or nearly complete occlusion of a main coronary artery, and 4 had healed infarcts: 45 had "much atheroma". Out of 106 patients, 61 had "non-specific" changes in the electrocardiogram, and they comment that it was impossible to distinguish the minor changes of true myocardial ischaemia.

The abnormalities of the $U$ wave and Q- $U$ interval found in the present case may prove to have shown that they are not exclusively found with intracardiac lesions, though it then remains to be seen how far they are specific to subarachnoid haemorrhage (or other cerebrovascular lesions). Routine electrocardiographic studies in subarachnoid haemorrhage, combined with detailed myocardial histology whenever possible, may help to answer this question. This problem is now of much more than academic importance, since victims of subarachnoid haemorrhage and other cerebrovascular lesions are considered potential emergency donor material for cardiac transplants, and their suitability must at least depend on the certain exclusion of concomitant myocardial lesions.

\section{Summary}

A case of subarachnoid haemorrhage is described in which the electrocardiogram showed independent deeply inverted $U$ waves, combined with a much prolonged $\mathrm{Q}-\mathrm{U}$ interval. It is suggested that this abnormality may prove to be diagnostic of subarachnoid haemorrhage, uncomplicated by myocardial disease, and as these findings have not been reported previously, they should be sought for in all cases of this lesion in order to determine their specificity.

I am most grateful to Dr. T. M. Dauncey for the pathological investigations in this case.

\section{References}

Burch, G. E., Meyers, R., and Abildskov, J. A. (1954). A new ECG pattern observed in cerebrovascular accidents. Circulation, 9, 719.

Furbetta, D., Bufalari, A., Santucci, F., and Solinas, P. (1956). Abnormality of the $U$ wave and of the T-U segment of the ECG. The syndrome of the papillary muscles. Circulation, 14, 1129.

Hoffbrand, B. I., and Morgan, B. D. G. (1965). Functional significance of electrocardiographic changes associated with subarachnoid haemorrhage. Lancet, 1, 844.

Hugenholtz, P. G. (1962). ECG abnormalities in cerebral disorders. Amer. Heart $\mathcal{F} ., 63,451$.

Koskelo, P., Punsar, S., and Sipila, W. (1964). Subendocardial haemorrhage and ECG changes in intracranial bleeding. Brit. med. F., 1, 1479.

Lepeschkin, E. (1955). The U wave of the ECG. Arch. intern. Med., 96, 600 .

Papp, C. (1940). U, the sixth wave of the electrocardiogram. Brit. Heart f., $2,9$.

Sarner, M., and Crawford, M. D. (1965). Ruptured intracranial aneurysm. Lancet, $2,1251$.

Shuster, S. (1960). The electrocardiogram in subarachnoid haemorrhage. Brit. Heart f., 22, 316.

Srivastava, S. C., and Robson, A. O. (1964). Electrocardiographic abnormalities associated with subarachnoid haemorrhage. Lancet, 2, 431.

Wasserman, F., Choquette, G., Casinelli, R., and Bellet, S. (1956). ECG observations in patients with cerebrovascular accidents. Amer. F. med. Sci., 231, 502. 